\title{
Intraoperative arteriovenous malformation rupture: causes, management techniques, outcomes, and the effect of neurosurgeon experience
}

\author{
Ramon Torné, M.D., ${ }^{1}$ Ana Rodríguez-Hernández, M.D., ${ }^{1}$ and Michael T. Lawton, M.D. ${ }^{2}$ \\ ${ }^{1}$ Department of Neurological Surgery, Vall d'Hebron Hospital, Universitat Autònoma de Barcelona, Spain; \\ and ${ }^{2}$ Department of Neurological Surgery, University of California, San Francisco, California
}

\begin{abstract}
Object. Intraoperative rupture can transform an arteriovenous malformation (AVM) resection. Blood suffuses the field and visualization is lost; suction must clear the field and the hand holding the suction device is immobilized; the resection stalls while hemostasis is being reestablished; the cause and site of the bleeding may be unclear; bleeding may force technical errors and morbidity from chasing the source into eloquent white matter; and AVM bleeding can be so brisk that it overwhelms the neurosurgeon. The authors reviewed their experience with this dangerous complication to examine its causes, management, and outcomes.

Methods. From a cohort of 591 patients with AVMs treated surgically during a 15-year period, 32 patients (5\%) experienced intraoperative AVM rupture. Their prospective data and medical records were reviewed.

Results. Intraoperative AVM rupture was not correlated with presenting hemorrhage, but had a slightly higher incidence infratentorially (7\%) than supratentorially (5\%). Rupture was due to arterial bleeding in 18 patients $(56 \%)$, premature occlusion of a major draining vein in $10(31 \%)$, and nidal penetration in $4(13 \%)$. In 14 cases $(44 \%)$, bleeding control was abandoned and the AVM was removed immediately ("commando resection"). The incidence of intraoperative rupture was highest during the initial 5-year period (9\%) and dropped to $3 \%$ and $4 \%$ in the second and third 5 -year periods, respectively. Ruptures due to premature venous occlusion and nidal penetration diminished with experience, whereas those due to arterial bleeding remained steady. Despite intraoperative rupture, $90 \%$ of AVMs were completely resected initially and all of them ultimately. Intraoperative rupture negatively impacted outcome, with significantly higher final modified Rankin Scale scores (mean 2.8) than in the overall cohort (mean 1.5; p < 0.001).

Conclusions. Intraoperative AVM rupture is an uncommon complication caused by pathological arterial anatomy and by technical mistakes in judging the dissection distance from the AVM margin and in mishandling or misinterpreting the draining veins. The decrease in intraoperative rupture rate over time suggests the existence of a learning curve. In contrast, intraoperative rupture due to arterial bleeding reflects the difficulty with dysplastic feeding vessels and deep perforator anatomy rather than neurosurgeon experience. The results demonstrate that intraoperative AVM rupture negatively impacts patient outcome, and that skills in managing this catastrophe are critical. (http://thejns.org/doi/abs/10.3171/2014.6.FOCUS14218)
\end{abstract} KEY WORDS $\bullet$ arteriovenous malformation $\bullet \quad$ intraoperative rupture
microsurgical resection $\bullet$ technique $\bullet$ learning curve

A RTERIOVENOUS malformation (AVM) resection should be bloodless, with sharp dissection of subarachnoid spaces, precise occlusion of pial feeding vessels, clean dissection of parenchymal margins, and careful occlusion of deep supply along the ependymal plane. ${ }^{1,2,6,9,14,15}$ The slightest bleeding is meticulously stopped to maintain absolute hemostasis before progressing to the next step. Ironically, AVM resections are some of the cleanest and driest dissections despite their highly charged hemodynamics. Nonetheless, some intraoperative bleeding is inevitable with AVM resection, no matter how meticulous the dissection, how skilled the neurosur-

Abbreviations used in this paper: $\mathrm{AVM}=$ arteriovenous malformation; $\mathrm{mRS}=$ modified Rankin Scale. geon, or how cooperative the AVM. Cauterizing dysplastic arteries with high flow will stir up bleeding that ranges from a small trickle from a pesky perforator to a torrent from nidal rupture. The threat of intraoperative rupture is pervasive in these cases, and when it occurs, it can transform the operation. Blood suffuses the field and visualization is lost; suction must remain on the bleeding vessel to clear the field and the hand holding the suction device is immobilized; the resection stalls while hemostasis is being reestablished; the cause and site of the bleeding may be unclear; bleeding may force technical errors and morbidity from chasing the source into eloquent white matter; and AVM bleeding can be so brisk that it overwhelms the neurosurgeon and spirals out of control. Although AVM bleeding is unnerving, it must be met with a swift response to avert catastrophe. 
The technical response to intraoperative AVM rupture differs from aneurysm rupture, which progresses through an orderly sequence of tamponade, suction, proximal control with temporary clipping, distal control with temporary clipping, and permanent aneurysm clipping. There is no aneurysm dome to tamponade with AVM bleeding, and there are too many feeding arteries for proximal control. There is no temporary clipping or neck to close. The bleeding source must be pursued, exposed, and controlled. A small cottonoid is applied at or near the bleeding site and suctioned to dry the field. A jet of blood is traced back to the source, which may be arterial, venous, or nidal. Although there is an extensive literature about intraoperative aneurysm rupture, its management, and the effects on patient outcome, relatively little has been written about intraoperative AVM rupture. ${ }^{5}$ Therefore, we reviewed our experience with this feared complication to examine these same factors.

\section{Methods}

\section{Patient Population}

The study was approved by the Committee on Human Research and conducted in compliance with Health Insurance Portability and Accountability Act regulations. Overall, 591 patients with AVMs were treated surgically by the senior author (M.T.L.) during a 15-year period between September 1997 and January 2013, including 297 female and 294 male patients with a mean age of 38 years (range 1-90 years). Operative reports and surgeon records were reviewed to identify 32 patients $(5 \%)$ experiencing intraoperative AVM rupture. Their inpatient charts, imaging studies, outpatient clinic evaluations, and data from the prospectively maintained database of the vascular neurosurgery service were reviewed to analyze the patient demographics, AVM features, and surgical factors associated with such events.

\section{Intraoperative AVM Rupture}

Intraoperative AVM rupture was defined as torrential bleeding from the AVM, above and beyond what is encountered in typical resections, that was difficult to control. Ruptures were further categorized as follows: 1) bleeding from ruptured or escaped feeding arteries at the AVM margin; 2) bleeding from a microsurgical violation of the AVM margin with penetration of the nidus; and 3) distension and bursting of the nidus due to premature occlusion of venous outflow.

\section{Postoperative Outcomes}

Radiographic outcome was assessed with postoperative angiography performed in all patients after microsurgical resection. Patient outcome was assessed using modified Rankin Scale (mRS) scores. Neurological evaluations were performed by a neurologist, neurosurgeon, or research nurse during postoperative clinic visits. Good outcomes were defined as final mRS scores of $0-2$, and poor outcomes were defined as a final mRS scores $>2$. Improvement was defined as a decrease in mRS score, and deterioration was defined as an increase in $\mathrm{mRS}$ score.

\section{Results}

\section{Patient Demographic Data}

There was a male predominance among the 32 patients with intraoperative AVM rupture (21 male patients, $66 \%$ ), and the mean age was higher than the age of the overall group (43 years, range 11-90 years). Patients with intraoperative AVM rupture were not more likely to present with hemorrhage (17 patients, 53\%, with hemorrhagic presentation). Two patients had incompletely obliterated AVMs after radiosurgery, and 11 patients (34\%) underwent preoperative embolization (Table 1). Five patients (16\%) had flow-related aneurysms, but these were not the source of intraoperative rupture.

\section{Characteristics of the AVMs}

Intraoperative rupture was associated with AVMs in all locations (Table 1). Twenty-five of the intraoperative ruptures $(78 \%)$ occurred with supratentorial AVMs and 7 (22\%) occurred with infratentorial AVMs. However, intraoperative rupture had a slightly higher incidence infratentorially (7 [7\%] of 95 AVMs) than supratentorially (25 [5\%] of 496 AVMs), although this was not statistically significant $(\mathrm{p}=0.36)$.

The majority of intraoperative ruptures occurred with AVMs of intermediate (44\%) and high (31\%) Spetzler-Martin $\operatorname{grades}^{20}$ (Table 2). Size was an important point, with 22 AVMs $(69 \%)$ measuring greater than 3 $\mathrm{cm}$ in diameter. Similarly, the majority of intraoperative ruptures occurred with AVMs of intermediate (19\%) and high (66\%) supplementary grades..$^{12}$ Of the factors incorporated into the supplementary grade, ${ }^{3,4,8,12}$ diffuseness was an important point, with 20 patients (63\%) having diffuse AVMs, which is approximately twice the overall incidence of diffuse AVMs.

\section{Intraoperative AVM Rupture}

Arterial bleeding was the most common type of intraoperative AVM rupture; it was observed in 18 patients $(56 \%)$. Furthermore, the majority of these arterial bleeding events were associated with deep perforating feeding vessels traversing white matter tracts to supply the deep plane of the nidus; such vessels are often difficult to visualize and difficult to coagulate. Frank AVM rupture occurred in 10 patients $(31 \%)$ after premature occlusion of a major draining vein. Inadvertent nidal penetration was the cause of intraoperative rupture in 4 patients (13\%). In 18 cases $(56 \%)$, intraoperative rupture was managed by controlling the bleeding or working through the bleeding to finish the resection quickly. In the remaining 14 cases (44\%), control of bleeding was considered hopeless, as was the ability to work through the bleeding, and the dissection plan was changed to remove the AVM immediately. This so-called commando resection opened access to deep anatomy to control bleeding points or disconnect the final feeding vessels. Eleven AVMs (79\%) requiring commando resection were greater than $3 \mathrm{~cm}$ in size and 11 were diffuse. Six (33\%) of 18 cases of uncontrolled arterial bleeding required commando resection, whereas 6 $(60 \%)$ of 10 cases of frank rupture after premature venous occlusion required commando resection. 
Intraoperative arteriovenous malformation rupture

TABLE 1: Characteristics of patients and AVMs, and registered cause of intraoperative rupture*

\begin{tabular}{|c|c|c|c|c|c|c|c|c|c|}
\hline \multirow[b]{2}{*}{ Case No. } & \multirow[b]{2}{*}{ Age (yrs), Sex } & \multirow[b]{2}{*}{ AVM Location } & \multicolumn{2}{|c|}{ AVM Grade } & \multicolumn{2}{|c|}{ Previous Treatments } & \multicolumn{3}{|c|}{ Cause of Intraop Rupture } \\
\hline & & & $\mathrm{SM}$ & Suppl & Radiosurgery & Embolization & Arterial & Nidal & Venous \\
\hline 1 & $41, \mathrm{~F}$ & parietal & 4 & 4 & yes & no & no & yes & no \\
\hline 2 & $25, \mathrm{~F}$ & frontal & 3 & 3 & no & no & no & no & yes \\
\hline 3 & $67, \mathrm{M}$ & cerebellar & 3 & 4 & no & no & no & no & yes \\
\hline 4 & $40, M$ & frontal & 1 & 2 & no & no & no & no & yes \\
\hline 5 & $15, M$ & occipital & 4 & 2 & no & yes & no & no & yes \\
\hline 6 & $33, \mathrm{M}$ & temporal & 4 & 2 & no & yes & yes & no & no \\
\hline 7 & $63, M$ & cerebellar & 3 & 4 & no & no & yes & no & no \\
\hline 8 & $46, M$ & occipital & 3 & 4 & no & yes & yes & no & no \\
\hline 9 & $46, F$ & occipital & 2 & 4 & no & no & yes & no & no \\
\hline 10 & $34, \mathrm{M}$ & cerebellar & 4 & 4 & no & yes & yes & no & no \\
\hline 11 & $68, F$ & deep central & 2 & 4 & no & no & yes & no & no \\
\hline 12 & $31, \mathrm{~F}$ & frontal & 2 & 2 & no & yes & yes & no & no \\
\hline 13 & $75, \mathrm{M}$ & brainstem & 3 & 3 & no & no & no & no & yes \\
\hline 14 & $11, \mathrm{M}$ & cerebellar & 3 & 3 & no & no & yes & no & no \\
\hline 15 & $46, M$ & cerebellar & 3 & 4 & no & yes & yes & no & no \\
\hline 16 & $37, \mathrm{~F}$ & parietal & 5 & 3 & no & no & yes & no & no \\
\hline 17 & $69, \mathrm{M}$ & parietal & 3 & 4 & no & no & no & no & yes \\
\hline 18 & $41, \mathrm{M}$ & parietal & 3 & 4 & no & no & yes & no & no \\
\hline 19 & $40, \mathrm{~F}$ & frontal & 3 & 4 & yes & no & no & yes & no \\
\hline 20 & $23, \mathrm{M}$ & frontal & 4 & 3 & no & no & yes & no & no \\
\hline 21 & $31, \mathrm{~F}$ & temporal & 3 & 4 & no & no & no & yes & no \\
\hline 22 & $26, \mathrm{M}$ & occipital & 5 & 4 & no & yes & no & no & yes \\
\hline 23 & $46, F$ & temporal & 2 & 4 & no & no & yes & no & no \\
\hline 24 & $37, M$ & temporal & 1 & 4 & no & no & no & no & yes \\
\hline 25 & $45, M$ & temporal & 3 & 3 & no & yes & yes & no & no \\
\hline 26 & $41, \mathrm{M}$ & parietal & 4 & 4 & no & no & no & yes & no \\
\hline 27 & $48, \mathrm{~F}$ & frontal & 4 & 4 & no & yes & no & no & yes \\
\hline 28 & $61, M$ & temporal & 2 & 4 & no & no & yes & no & no \\
\hline 29 & $65, M$ & cerebellar & 3 & 4 & no & no & yes & no & no \\
\hline 30 & $20, \mathrm{M}$ & temporal & 4 & 2 & no & yes & yes & no & no \\
\hline 31 & $90, \mathrm{~F}$ & temporal & 1 & 4 & no & no & yes & no & no \\
\hline 32 & $36, \mathrm{M}$ & parietal & 3 & 4 & no & yes & no & no & yes \\
\hline
\end{tabular}

* SM = Spetzler-Martin; Suppl = supplementary.

More than half of the intraoperative ruptures (17 cases, 53\%) happened during the first 5 years of the senior author's experience. The incidence of intraoperative rupture was highest during this 5-year period (17 ruptures [9\%] of 180 microsurgical resections), dropping to $3 \%$ (5 ruptures in 177 resections) in the second 5-year period and 4\% (10 ruptures in 234 resections) in the third 5-year period. This decrease in rupture rate from the first period to subsequent periods was statistically significant $(\mathrm{p}=$ 0.037 ). Most of the ruptures caused by premature venous occlusion occurred during this initial 5-year experience (7 [70\%] of 10 cases), and all 4 of the ruptures caused by nidal penetration occurred then, indicating that these technical mistakes may be corrected by experience. Intraoperative rupture due to arterial bleeding was more evenly distributed (6 of 18 during the initial 5-year period), in- dicating that the difficulty in managing deep perforating vessels is determined more by anatomy than surgeon experience. Commando resections were also more frequent during the initial 5-year period (9 [53\%] of 17 cases).

Interestingly, both patients previously treated radiosurgically experienced rupture from nidal penetration, indicating the difficulty in determining the nidal margin in these altered AVMs.

\section{Postoperative Outcomes}

Despite intraoperative rupture, $29(90 \%)$ of 32 AVMs were completely resected. In 3 patients $(10 \%)$, residual nidus was seen on postoperative angiography, and these individuals required a second surgical stage to remove the AVM completely (100\% complete resection rate). This incidence of residual AVM was higher than that in the 
TABLE 2: Summary of AVM grades in 32 patients

\begin{tabular}{cc}
\hline AVM Grade & No. $(\%)$ \\
\hline Spetzler-Martin & \\
1 & $3(9.4)$ \\
2 & $5(15.6)$ \\
3 & $14(43.8)$ \\
4 & $8(25.0)$ \\
5 & $2(6.3)$ \\
Supplementary & \\
1 & $0(0)$ \\
2 & $5(15.6)$ \\
3 & $6(18.8)$ \\
4 & $21(65.6)$ \\
5 & $0(0)$ \\
\hline
\end{tabular}

overall experience (22 [4\%] of 591 patients), indicating that complete resection may be compromised when surgery is complicated by intraoperative rupture.

Four patients died in the perioperative period (surgical mortality $12.5 \%)$ and 4 more patients $(12.5 \%)$ died at late follow-up (Table 3). Eighteen patients (56\%) were improved or unchanged relative to their preoperative $\mathrm{mRS}$ score, and 6 patients $(19 \%)$ were worse (mean follow-up duration 10 months). Of these 6 patients, 4 still experienced good outcomes ( $\mathrm{mRS} \geq 2$ ). Outcomes improved in later 5-year periods compared with the first period. Still, intraoperative AVM rupture negatively impacted out- come, with significantly higher final $\mathrm{mRS}$ scores (mean 2.8 and median 3) than in the overall cohort (mean 1.5 and median $1 ; \mathrm{p}<0.001)$.

\section{Discussion}

Intraoperative AVM rupture is an uncommon complication, occurring with a 5\% frequency that is slightly less than intraoperative aneurysm rupture (7\%-15\% in our experience $\left.{ }^{16,19}\right)$. Unlike aneurysms that are fragile when they present with subarachnoid hemorrhage and are more likely to rerupture during dissection, intraoperative AVM rupture was not correlated with hemorrhagic presentation. ${ }^{11}$ It appears to be caused by technical mistakes in judging the dissection distance from the AVM margin and in mishandling or misinterpreting the draining veins. The drop in intraoperative rupture rate over time and in these two causes in particular suggests the existence of a learning curve, as with most challenging neurosurgical operations. ${ }^{21}$ In contrast, the intraoperative rupture rate due to arterial bleeding was steady throughout the study period, indicating that the difficulty with dysplastic feeding and deep perforating vessels reflects nasty anatomical challenges rather than neurosurgeon experience. Our experience demonstrated that intraoperative AVM rupture negatively impacted patient outcome, and therefore it is important to describe insights and techniques for managing this catastrophe that might help others progress through their learning curves.

\section{Intraoperative Rupture Due to Arterial Bleeding}

Arterial bleeding originates from the parenchymal

TABLE 3: Summary of clinical outcomes in 32 patients with intraoperative AVM rupture

\begin{tabular}{|c|c|c|c|c|c|c|c|c|}
\hline \multirow[b]{2}{*}{ Scores \& Outcomes } & \multicolumn{2}{|c|}{ Total } & \multicolumn{2}{|c|}{ Arterial Bleeding } & \multicolumn{2}{|c|}{ Nidal Penetration } & \multicolumn{2}{|c|}{ Venous Occlusion } \\
\hline & No. & $\%$ & No. & $\%$ & No. & $\%$ & No. & $\%$ \\
\hline \multicolumn{9}{|l|}{ preop mRS score } \\
\hline 0 & 4 & 12.5 & 2 & 6.2 & 1 & 3.1 & 1 & 3.1 \\
\hline 1 & 8 & 25 & 5 & 15.6 & 1 & 3.1 & 2 & 6.2 \\
\hline 2 & 5 & 15.6 & 3 & 9.4 & 0 & 0 & 2 & 6.2 \\
\hline 3 & 4 & 12.5 & 2 & 6.2 & 0 & 0 & 2 & 6.2 \\
\hline 4 & 8 & 25 & 4 & 12.5 & 2 & 6.2 & 2 & 6.2 \\
\hline 5 & 3 & 9.4 & 2 & 6.2 & 0 & 0 & 1 & 3.1 \\
\hline 6 & 0 & 0 & 0 & 0 & 0 & 0 & 0 & 0 \\
\hline \multicolumn{9}{|l|}{ final $m R S$ score } \\
\hline 0 & 5 & 15.6 & 2 & 6.2 & 1 & 3.1 & 2 & 6.2 \\
\hline 1 & 6 & 18.8 & 5 & 15.6 & 1 & 3.1 & 0 & 0 \\
\hline 2 & 4 & 12.5 & 2 & 6.2 & 0 & 0 & 2 & 6.2 \\
\hline 3 & 8 & 25 & 3 & 9.4 & 0 & 0 & 5 & 15.6 \\
\hline 4 & 1 & 3.1 & 1 & 3.1 & 0 & 0 & 0 & 0 \\
\hline 5 & 0 & 0 & 0 & 0 & 0 & 0 & 0 & 0 \\
\hline 6 & 8 & 25 & 5 & 15.6 & 2 & 6.2 & 1 & 3.1 \\
\hline \multicolumn{9}{|l|}{ relative outcome } \\
\hline improved & 11 & 34.4 & 8 & 25 & 0 & 0 & 3 & 9.4 \\
\hline unchanged & 7 & 21.9 & 2 & 6.2 & 2 & 6.2 & 3 & 9.4 \\
\hline worse & 6 & 18.8 & 3 & 9.4 & 0 & 0 & 3 & 9.4 \\
\hline dead & 8 & 25 & 5 & 15.6 & 2 & 6.2 & 1 & 3.1 \\
\hline
\end{tabular}


side of the resection bed, not from the nidus itself. A cottonoid prevents suction from injuring the brain, and some retraction pressure can be applied to the cottonoid with the sucker tip, allowing the sucker to be a dynamic retractor in the hunt. Small bleeding vessels can be controlled with bipolar forceps and suction, suctioning right at the bleeding point or even drawing the artery into the barrel of the sucker. The sucker simultaneously clears the blood and immobilizes the bleeder, while the bipolar cauterizes the artery proximally. When cautery fails, microclips will close small perforating arteries and aneurysm clips will control large feeding arteries. ${ }^{1,13}$ Thin, friable arteries become more manageable as they are exposed proximally. Therefore, arteries with intractable bleeding are chased proximally, unfortunately with deeper transgression into brain tissue that may jeopardize eloquent tracts or cortex. ${ }^{5,13}$

Arterial bleeders are points of hemorrhage that can be closed precisely. They should never be packed, covered with hemostatic agents, or left unattended because they will continue to bleed out of view, either intraparenchymally or intraventricularly. Concealed hemorrhages can also occur with arteries that reopen after they have been coagulated, cut, and retracted into brain parenchyma. These hemorrhages blossom into hematomas and fill ventricles without any apparent bleeding in the surgical field. Progressive brain fullness, narrowing of the surgical corridor, or outward herniation of brain may be the only signs of active bleeding. The hematoma may suddenly erupt into the surgical field. When there is an unexplained change in operative conditions and a concealed hemorrhage is suspected, areas with previous bleeding, persistent oozing, or friable arteries should be reexplored. A trail of edematous or hemorrhagic brain can often be followed to a clot, which is then evacuated and explored for the bleeder. Concealed ventricular hemorrhage quickly fills the ventricles and herniates brain outward, demanding aggressive entry into the ventricle to control the bleeder.

\section{Intraoperative Rupture Due to Nidal Penetration}

Dissecting too close to the AVM and violating its margin can rupture the nidus, ${ }^{22}$ although this was our least common cause. Judging the right dissection distance is an ongoing process that must balance the eloquence of the adjacent parenchyma with the dangers of dissecting close to the nidal margin. This process can be difficult with diffuse AVMs and those treated radiosurgically with altered margins. ${ }^{5,12,17}$ Small breaches are sometimes controlled with cautery, but it can also widen the hole and aggravate bleeding. A small piece of Nu-knit and gentle pressure with the sucker will control minor bleeding. For more significant bleeding, a fixed retractor blade on the Nu-knit will free the hands to continue operating. This hemostatic retraction applies pressure, buys time, and even advances circumdissection by lifting the nidus. The retractor is released after the bleeding site has clotted. Retracting an AVM can, by itself, result in AVM bleeding if it is excessive or if the blade sticks to the AVM margin. Therefore, Nu-knit is used to pad the blade and retraction pressure is minimized.

\section{Intraoperative Rupture Due to Venous Occlusion}

Frank AVM rupture usually occurs after inadver- tently occluding an AVM's venous outflow.,10,18 A large red vessel is eagerly cauterized and cut, expecting drastic reduction in the AVM's arterial supply. Instead, the AVM becomes tense with increased intranidal pressure, feeding arteries become difficult to coagulate, and the nidal margins pop with scattered points of hemorrhage remote from operative site. That misinterpreted "artery" was actually a major draining vein and the AVM is now on the verge of rupture. It is no longer possible to meticulously dissect the remaining planes or to control these random bleeding sites around the nidus. Instead, a commando resection must deliver the AVM before it erupts. There is an interval between the venous occlusion and rupture that is variable and unpredictable, and therefore the resection must accelerate. The occluded vein is no longer functional and can be divided to mobilize the AVM. The AVM is aggressively retracted to hunt down the remaining feeding arteries. Active bleeding requires a large sucker to maintain visualization, and excess bleeding is allowed to run out of the bed, rather than down into the bed. Attention is directed to larger, coagulable feeding vessels. Delicate feeders that would otherwise be carefully closed with microclips may have to wait until after the AVM is removed. Typically, there are two or three last feeding vessels on the polar opposite side of the nidus, and they are found by mobilizing the nidus out of the resection bed, even as the blood pools in it. After occluding these feeding vessels, the AVM slackens. Removal of the AVM opens the bed and makes it easier to scour for active bleeders, typically the smaller ones that were ignored.

The commando resection is a last resort when faced with impending or frank AVM rupture. It is not meant for simple arterial or venous bleeding, or for minor nidal bleeding. Rupture of an AVM calls for decisive action, and the commando resection represents a point of no return. It is a test of one's mettle, requiring the recognition of a mistake and the courage to battle on. There are few situations in neurosurgery more challenging than this one. One must summon skill, insight, and grit at the end of a long case. One must quiet the rush of fear, confusion, regret, stress, frustration, and excitement. One must find calm, clarity, and confidence to continue operating. One must instruct nurses passing instruments, neurosurgeons providing assistance, and anesthesiologists replenishing the blood loss. Although the orderly rhythm of a meticulous AVM resection is suddenly replaced with frenzied chaos, it need not shake one's confidence. All of the AVMs in this experience were resected and no patient died on the operating table. Getting through an intraoperative AVM rupture and a commando resection is an important milestone. It is inevitable for the busy AVM surgeon; it exposes technical errors that can be rectified, like misinterpreting red veins; it establishes one's ability to manage a disaster; and it builds confidence for all subsequent AVM resections, knowing that even the worst conditions are manageable. In the end, it's just bleeding and one's ability to keep working despite bleeding. While the amount of bleeding in these explosive situations can be shocking, it can also be ignored, fixating instead on the last arterial targets and completion of the surgical plan. 


\section{Conclusions}

Intraoperative AVM rupture is an uncommon complication caused by pathological arterial anatomy and by technical mistakes in judging the dissection distance from the AVM margin and in mishandling or misinterpreting the draining veins. The decrease in intraoperative rupture rate over time suggests the existence of a learning curve. In contrast, intraoperative rupture due to arterial bleeding reflects the difficulty with dysplastic feeding vessels and deep perforator anatomy rather than neurosurgeon experience. Our results demonstrate that intraoperative AVM rupture negatively impacts patient outcome, and that skills in managing this catastrophe are critical.

\section{Acknowledgment}

Dr. Torné was supported for this research project by a grant from the Cerebrovascular Section of the Spanish Neurosurgical Society.

\section{Disclosure}

The authors report no conflict of interest concerning the materials or methods used in this study or the findings specified in this paper.

Author contributions to the study and manuscript preparation include the following. Conception and design: Lawton. Acquisition of data: all authors. Analysis and interpretation of data: all authors. Drafting the article: all authors. Critically revising the article: all authors. Reviewed submitted version of manuscript: all authors. Approved the final version of the manuscript on behalf of all authors: Rodríguez-Hernández. Statistical analysis: Torné. Study supervision: Lawton.

\section{References}

1. Bendok BR, El Tecle NE, El Ahmadieh TY, Koht A, Gallagher TA, Carroll TJ, et al: Advances and innovations in brain arteriovenous malformation surgery. Neurosurgery 74 (Suppl 1):S60-S73, 2014

2. Clatterbuck RE, Hsu FPK, Spetzler RF: Supratentorial arteriovenous malformations. Neurosurgery 57 (1 Suppl):164-167, 2005

3. Davies JM, Kim H, Young WL, Lawton MT: Classification schemes for arteriovenous malformations. Neurosurg Clin $\mathbf{N}$ Am 23:43-53, 2012

4. Ding D, Liu KC: Predictive capability of the Spetzler-Martin versus supplementary grading scale for microsurgical outcomes of cerebellar arteriovenous malformations. J Cerebrovasc Endovasc Neurosurg 15:307-310, 2013

5. Du R, Keyoung HM, Dowd CF, Young WL, Lawton MT: The effects of diffuseness and deep perforating artery supply on outcomes after microsurgical resection of brain arteriovenous malformations. Neurosurgery 60:638-648, 2007

6. Gabarrós Canals A, Rodríguez-Hernández A, Young WL, Lawton MT, UCSF Brain AVM Study Project: Temporal lobe arteriovenous malformations: anatomical subtypes, surgical strategy, and outcomes. Clinical article. J Neurosurg 119:616-628, 2013

7. Hademenos GJ, Massoud TF: Risk of intracranial arteriovenous malformation rupture due to venous drainage impairment. A theoretical analysis. Stroke 27:1072-1083, 1996

8. Kim H, Pourmohamad T, Westbroek EM, McCulloch CE, Lawton MT, Young WL: Evaluating performance of the SpetzlerMartin supplemented model in selecting patients with brain arteriovenous malformation for surgery. Stroke 43:2497-2499, 2012

9. Kim YB, Young WL, Lawton MT: Parafalcine and midline arteriovenous malformations: surgical strategy, techniques, and outcomes. Clinical article. J Neurosurg 114:984-993, 2011

10. Lane BC, Cohen-Gadol AA: A prospective study of microscope-integrated intraoperative fluorescein videoangiography during arteriovenous malformation surgery: preliminary results. Neurosurg Focus 36(2):E15, 2014

11. Lawton MT, Du R, Tran MN, Achrol AS, McCulloch CE, Johnston SC, et al: Effect of presenting hemorrhage on outcome after microsurgical resection of brain arteriovenous malformations. Neurosurgery 56:485-493, 2005

12. Lawton MT, Kim H, McCulloch CE, Mikhak B, Young WL: A supplementary grading scale for selecting patients with brain arteriovenous malformations for surgery. Neurosurgery 66:702-713, 2010

13. Morgan MK, Drummond KJ, Grinnell V, Sorby W: Surgery for cerebral arteriovenous malformation: risks related to lenticulostriate arterial supply. J Neurosurg 86:801-805, 1997

14. Potts MB, Young WL, Lawton MT, UCSF Brain AVM Study Project: Deep arteriovenous malformations in the Basal Ganglia, thalamus, and insula: microsurgical management, techniques, and results. Neurosurgery 73:417-429, 2013

15. Rodríguez-Hernández A, Kim H, Pourmohamad T, Young WL, Lawton MT: Cerebellar arteriovenous malformations: anatomic subtypes, surgical results, and increased predictive accuracy of the supplementary grading system. Neurosurgery 71:1111-1124, 2012

16. Sanai N, Caldwell N, Englot DJ, Lawton MT: Advanced technical skills are required for microsurgical clipping of posterior communicating artery aneurysms in the endovascular era. Neurosurgery 71:285-295, 2012

17. Sanchez-Mejia RO, McDermott MW, Tan J, Kim H, Young WL, Lawton MT: Radiosurgery facilitates resection of brain arteriovenous malformations and reduces surgical morbidity. Neurosurgery 64:231-240, 2009

18. Schaller C, Urbach H, Schramm J, Meyer B: Role of venous drainage in cerebral arteriovenous malformation surgery, as related to the development of postoperative hyperperfusion injury. Neurosurgery 51:921-929, 2002

19. Sheth SA, Hausrath D, Numis AL, Lawton MT, Josephson SA: Intraoperative rerupture during surgical treatment of aneurysmal subarachnoid hemorrhage is not associated with an increased risk of vasospasm. Clinical article. J Neurosurg 120:409-414, 2014

20. Spetzler RF, Martin NA: A proposed grading system for arteriovenous malformations. J Neurosurg 65:476-483, 1986

21. Vilalta J, Arikan F, Torné R: [The decalogue on the arteriovenous malformation.] Neurocirugia (Astur) 24:229-230, 2013 (Span)

22. Zaidi HA, Abla AA, Nakaji P, Chowdhry SA, Albuquerque FC, Spetzler RF: Indocyanine green angiography in the surgical management of cerebral arteriovenous malformations: lessons learned in 130 consecutive cases. Neurosurgery 10 (Suppl 2):246-251, 2014

Manuscript submitted May 15, 2014.

Accepted June 10, 2014.

Please include this information when citing this paper: DOI: 10.3171/2014.6.FOCUS14218.

Address correspondence to: Ana Rodríguez-Hernández, M.D., Department of Neurological Surgery, Vall d'Hebron Hospital, Universitat Autònoma de Barcelona, Pg. Vall d'Hebron 119-129, 08035 Barcelona, Spain. email: ana.neurosurgery@hotmail.com. 